\title{
Clustering and Energy Efficient Routing Protocol for Wireless Sensor Network using Genetic Algorithm
}

\author{
Amit Singh \\ Computer Science \\ Ajmer, India
}

\author{
Khushbu Babbar \\ Computer Science \\ Ajmer, India
}

\author{
Apeksha Malik \\ Computer Science \\ Ajmer, India
}

\begin{abstract}
Energy consumption is the most important issue in wireless sensor networks. In wireless senor network, node is battery driven so when the data is transferred or received by node energy is consumed and lifetime of node gets decreased. So in order to increase the network lifetime, we propose an energy efficient routing protocol for wireless sensor network for randomly deployed sensor nodes. The basic purpose of the project is to develop a new routing protocol for the Wireless sensor network (WSN) under hierarchical approach. A sensor network is composed of a large number of sensor nodes which consist of sensing power, computation and communication capabilities. The major entities in WSN are sensing node, base station, and end user. Clustering is used as key techniques in energy efficient routing protocol. Genetic algorithm is used for selection of cluster head. In this protocol multi hop communication is used between the cluster heads and cluster head to base station. For cluster head selection, each node calculates its fitness function which is based on distance, energy and probability and uses distribution function for crossover. MATLAB is used for the simulation. The routing protocol produces optimal cluster heads for wireless sensor network for the communication which results in nodes alive for the long time a comparison of algorithm with benchmark algorithms is also performed. Simulation results shows that propose algorithm increases the network lifetime
\end{abstract}

\section{Keywords}

Wireless sensor network, Energy, Base station, Cluster, Genetic algorithm

\section{INTRODUCTION}

A Wireless Sensor Networks (WSN) is a set of hundreds or thousands of micro sensor nodes that have capacity of sensing and establishing wireless communication between nodes and doing computational and processing operations. A wireless sensor network consists of sensor nodes deployed over geographical area for monitoring physical phenomena like temperature, humidity, vibrations and so on. WSN consists of one or more base stations (or sinks) and a large number of sensor nodes which are used for monitoring or sensing data. Wireless nodes are smaller in size and power supply is limited which is provided by a small battery which cannot replaces or recharged frequently when deployed in a non-reachable places.

The rest of the paper is structured as follows. In section 2, we briefly review about the genetic algorithm. Section 3 provides brief description about network preliminaries. In section 4 proposed approaches is explained Section 5 describes the simulation parameters and assumptions made. Section 6 presents the experimental setup and simulation results. Section 7 concludes this paper by describing the summary of results obtained.

\section{GENETIC ALGORITHM}

Genetic algorithms are randomized search and optimization techniques guided by the principles of evolution and natural genetics. Initially, the genetic algorithm begins with a random population that consists of genes. In the next step, the algorithm lean individuals toward the optimum solution through repeated processes such as crossover and selection operators.

Steps used in proposed genetic algorithm are:

Begin

1. $\mathrm{t}=0$

2. Initialized population $\mathrm{P}(\mathrm{t})$

3. Computer fitness $\mathrm{P}(\mathrm{t})$

4. $\mathrm{t}=\mathrm{t}+1$

5. If termination criterion achieved go to step 10

6. Select $\mathrm{P}(\mathrm{t})$ from $\mathrm{P}(\mathrm{t}-1)$

7. Crossover $\mathrm{P}(\mathrm{t})$

8. Mutate $\mathrm{P}(\mathrm{t})$

9. Go to step 3

10. Output best and stop

End

\section{NETWORK PRELIMINARIES FOR THE PROPOSED WORK}

- Simulation is performed in MATLAB

- The network is homogenous and all nodes have same initial energy and probability $\mathrm{p}$.

- A network with 100 nodes deployed randomly over an area of size $100 \times 100 \mathrm{~m}^{2}$.

- All nodes consume same energy for transmission and reception.

- Data aggregation is done by the Cluster Head.

- A node, which has become cluster head, shall be eligible to become cluster head after $1 / p$ rounds.

- Energy of transmission is depends on the distance (source to destination). 


\section{PROPOSED APPROACH FOR CLUSTERING USING GENETIC ALGORITHM}

The methodology used for clustering and energy efficient routing protocol we used Genetic algorithm for selection of cluster head. In this protocol multi hop communication is used within cluster and cluster head to base station. For cluster head selection, each node calculates its fitness function which is based on energy, probability and distance between base station and node. Selected nodes send its information to base station. Base station performs the genetic algorithm to select the best cluster head and then non cluster head send their data to near cluster head and these cluster head forward data to their near head cluster head or base station which results in nodes alive for the long time. Proposed clustering and routing algorithm works in rounds. In this study, multi-hop communication is used within the normal nodes and from cluster head to BS. Each round consists two phases- Initial phase and Steady phase. In Initial phase all nodes send the residual energy, distance and probability parameters to the own fitness function. The nodes with maximum result of fitness function are choosed by the network administrator and notify its candidacy as cluster head to the BS. The aim of the project is to find the cluster heads with higher capabilities and distribute them in the network so that the total network energy consumption is minimized.

\subsection{Initial Phase}

In initial phase of each round a population and fitness function is be used to select cluster head and cluster member. In first round of algorithm choose $M$ initial cluster heads $\mathrm{a}_{1}$, $\mathrm{a}_{2} \ldots \ldots, \mathrm{a}_{\mathrm{M}}$ from the $\mathrm{n}$ nodes $\left\{\mathrm{y}_{1}, \mathrm{y}_{2}, \ldots \ldots, \mathrm{y}_{\mathrm{n}}\right\}$. Then cluster member will be selected for each cluster head.

Step1: At the beginning all nodes have same probability to become a cluster head.

Step2: $\mathrm{F}(\mathrm{x})$ is the fitness function used to select the cluster head and defined as

$$
\mathrm{F}(\mathrm{x})=(\mathrm{G}(\mathrm{n}) * \mathrm{E}(\mathrm{r}) * \mathrm{D})
$$

\section{Where}

$\mathrm{G}(\mathrm{n})=$ Probability of node to become cluster head as used in LEACH

$\mathrm{E}(\mathrm{r})=$ Node residual energy

$\mathrm{D}=$ Distance between node and base station

Step 3:

After calculating the built in $\mathrm{F}(\mathrm{x})$ function each node start a counter till predefined threshold value. For each network, network administrator decides number of cluster heads. The first node which reaches at a predefined threshold value will send its candidature to the base station for cluster head selection.

\section{Step 4: Selection of Cluster Head}

BS selects the cluster head and the result is sent to the network. At the base station selected number of nodes introduced themselves as the candidate of cluster head.

Single-point crossover is performed using a distribution function on candidate nodes which is selected and mutation is performed. After this base station selects a chromosome that has minimum difference of energy from last round and with best probability and high distribution function as cluster head. Other nodes tie to the closest $\mathrm{CH}$.

Step 5: BS sends the message to selected $\mathrm{CH}$

\section{Step 6: Cluster Formation}

Selected cluster head send advertisement to cluster nodes or normal nodes. Normal nodes join to closest cluster head to form cluster.

\subsection{Steady State Phase}

After initial state each node knows its $\mathrm{CH}$. Cluster head receive only one packet from member nodes of the cluster in each period. Cluster head aggregate and compress the data received from the attached normal node. After defining the head cluster in the initial phase, the multi-hop communication is used for transmission and the cluster head send controlled messages to the nearby cluster heads and the perfect path would be chosen. The perfect path is a way which the cluster head has more energy and closer distance to the base station and has no traffic. Then the data after being collected by the head cluster are sending to the same path and these would go on in the next cluster head until it is sent to the base station.

Step 1: Send a control message by cluster head to their surrounding cluster heads.

Step 2: The best head cluster is choose among all heads cluster which has best output for routing data.

Step 3: Check selected head cluster for traffic status and select other head cluster if traffic has happened.

Step 4: Send data to Specified head cluster.

Step 5: Execution steps 1 to 4 until the base station receive packets

\section{SIMULATION PARAMETERS}

Table 1: The values of genetic algorithm

\begin{tabular}{|c|c|}
\hline Parameter & Value \\
\hline Initial population & 100 \\
\hline Crossover rate & 0.5 \\
\hline Mutation rate & 0.5 \\
\hline
\end{tabular}


Table 2 Simulation parameter for Proposed Algorithm

\begin{tabular}{|l|l|}
\hline Parameter & Values \\
\hline Simulation Round & 8000 \\
\hline Number of nodes & $100+1$ (Node + BS) \\
\hline Topology Size & 100 X 100 \\
\hline Probability & 0.05 \\
\hline Initial Node Power & 0.5 Joule \\
\hline $\begin{array}{l}\text { Nodes Distribution } \\
\text { Energy for Transmission } \\
\text { (ETX) }\end{array}$ & $50 * 0.000000001$ \\
\hline $\begin{array}{l}\text { Energy for Reception (ERX) } \\
\text { Distributed }\end{array}$ & $50 * 0.000000001$ \\
\hline $\begin{array}{l}\text { Energy for Data } \\
\text { Aggregation (EDA) }\end{array}$ & $5 * 0.000000001$ \\
\hline
\end{tabular}

\section{SIMULATION RESULTS}

Simulation shows the following results in different scenario

\subsection{Comparison of LEACH and New Approach}

In this simulation result, the comparison is done between new approach and benchmark algorithm. In this the data is sends directly to base station which shows that the proposed algorithm increases the network lifetime by comparing with the LEACH protocol.

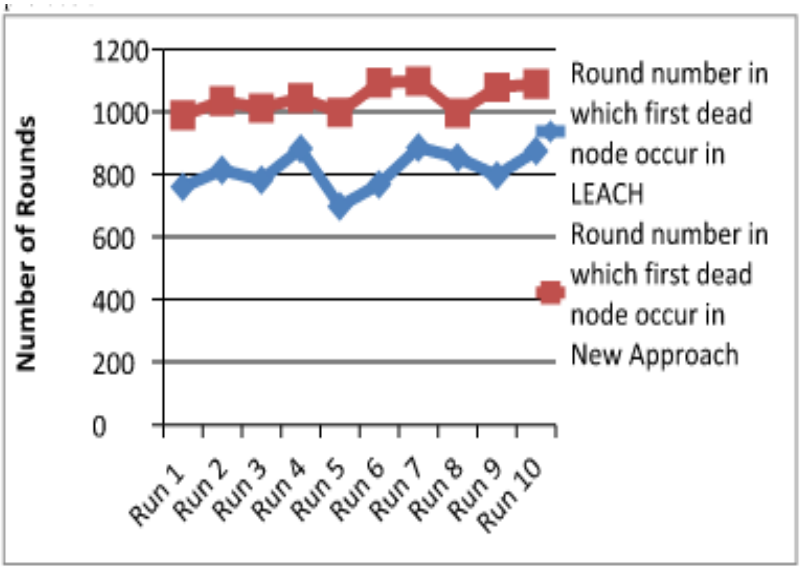

Fig 1. Number of Round in which first dead node occur

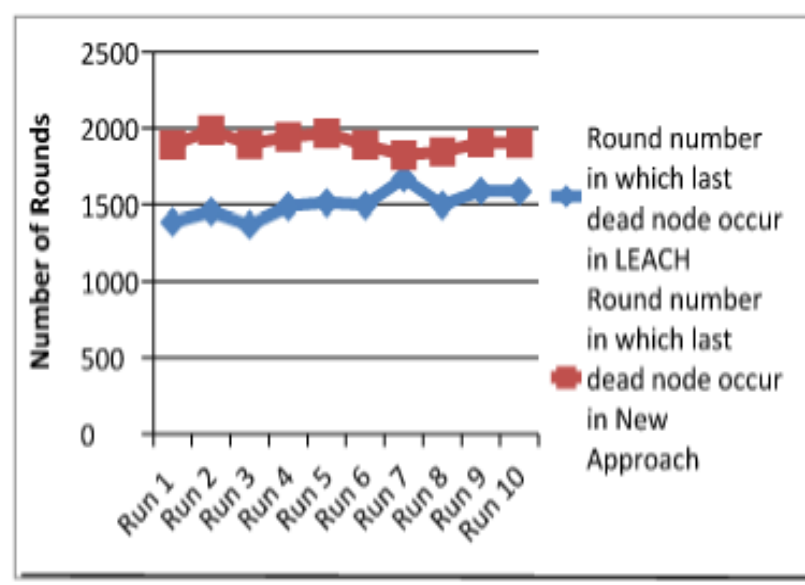

Fig 2: Number of round in which last dead node occur

\subsection{Round number in which dead node occur in New Approach}

In this simulation result, the comparison is done between two new approaches. First one the cluster head sends data directly to base station and second, the cluster head sends data to near cluster head instead directly to base station. In this round number is compared for first and last dead node for both the approaches which shows that by sending data to near cluster head instead of base station increases the network lifetime by consuming less energy.

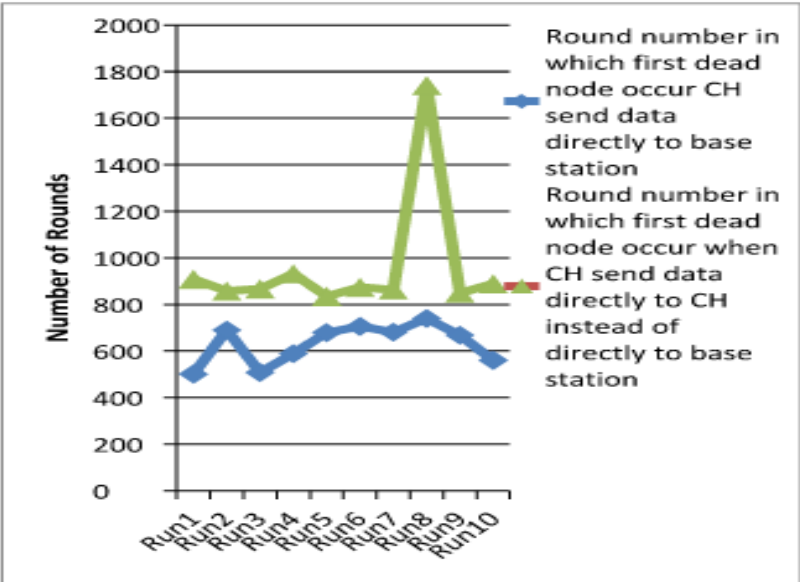

Fig 3. Number of Round in which first dead node occur in new approach

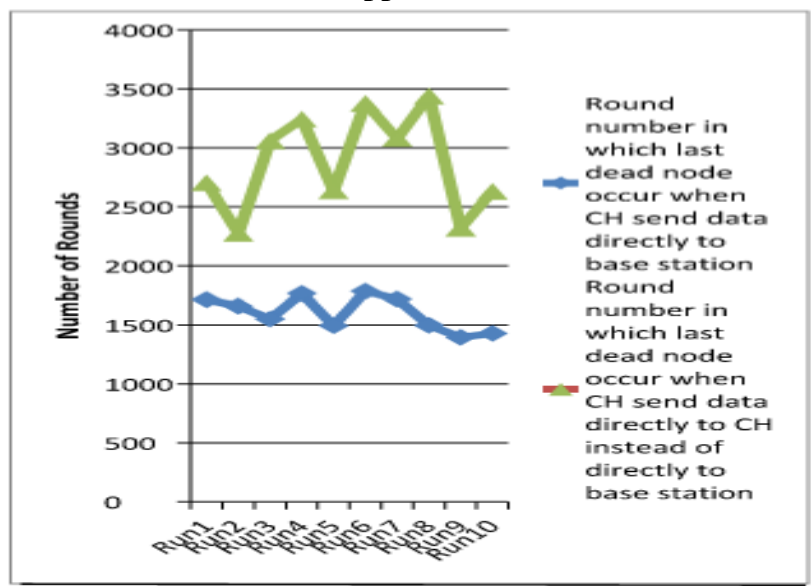

Fig4: Number of Round in which last dead node occur in new approach 


\section{CONCLUSION}

This paper presents the simulation result Table 8 shows the comparison and the number of rounds after which first dead node and last dead node occur in the network using LEACH and new approach. Table 9 shows the comparison and the number of rounds after which first dead node occur in the network when data sends directly to base station and data sends near $\mathrm{CH}$ instead of base station in new approach. The results show that the proposed approach outperforms LEACH terms of lifetime of network. We compared the LEACH protocol with the new technique under the same simulation condition and values. As results shows that the number of round in which first round dead is 734 from 10 simulations with LEACH protocol is increased to 1043 rounds with proposed algorithm. The proposed technique has improved the performance in terms of increasing the network lifetime by comparing with LEACH. Table 9 shows the results that the number of round in which first and last dead nodes when data is sends directly to base station are 1043 and 1904 is increased to 1861 and 2877 respectively when data is sends to near $\mathrm{CH}$ instead of base station.

\section{REFERENCES}

[1] K. Khamforoosh, and H. Khamforoush, "A new routing Algorithm for Energy Reduction in Wireless Sensor Networks", IEEE, 2009

[2] Jamal N. Al-Karaki , Ahmed E. Kamal " Routing Techniques in Wireless Sensor Networks: A Survey" IEEE Wireless Communications, Vol. 11, No. 6, pp.628, December 2004.

[3] M. Younis, M. Youssef and K. Arisha, "Energy-Aware Routing in Cluster-Based Sensor Networks", in the Proceedings of the 10th IEEE/ACM(MASCOTS2002), Fort Worth, TX, October 2002

[4] S. Lindsay and C. Raghavendra, "PEGASIS: PowerEfficient Gathering in Sensor Information Systems", international Conf. on Communications, 2001. Anew routing

[5] J.N Al-karaki et al.,"Data Agggregation in Wireless Sensor Networks - Exact and Approximate Algorithms," Proc. IEEE Wks. High Perf. Switching and Routing 2004, Phoenix, AZ, Apr. 18-21,2004.

[6] Gaurav Sharma, Suman Bala and A.K.Verma, "Routing Techniques in Wireless Sensor Networks: An Overview", International Conference on Intelligent Systems and Networks (IISN-09), Feb 14-18, 2009.

[7] J. Kulik, W. R. Heinzelman, and H. Balakrishnan, "Negotiation-based protocols for disseminating information in wireless sensor networks," Wireless Networks, Volume: 8, pp. 169-185, 2002.

[8] Manjeshwar and D. P. Agarwal, "TEEN: a routing protocol for enhanced efficiency in wireless sensor networks," In 1st International Workshop on Parallel and Distributed Computing Issues in Wireless Networks and Mobile Computing,April 2001.

[9] D. Braginsky and D. Estrin, IRumor Routing Algorithm for Sensor Networks," in the Proceedings of the First Workshop on Sensor Networks and Applications (WSNA), Atlanta, GA, October 2002 [10] F. Ye, A. Chen, S. Liu, L. Zhang, \A scalable solution to minimum cost forwarding in Communications and Networks (ICCCN), pp. 304-309, 2001.

[10] C. Schurgers and M.B. Srivastava, IEnergy efficient routing in wireless sensor networks", in the MILCOM Proceedings on Communications for Network-Centric Operations: Creating the Information Force, McLean, VA, 2001.

[11] M. Chu, H. Haussecker, and F. Zhao, IScalable Information-Driven Sensor Querying and Routing for ad hoc Heteroge-neous Sensor Networks," The International Journal of High Performance Computing Applications, Vol. 16, No. 3, August2002.

[12] Y. Yao and J. Gehrke, IThe cougar approach to innetwork query processing in sensor networks", in SIGMOD Record, September 2002.

[13] N. Sadagopan et al., The ACQUIRE mechanism for efficient querying in sensor networks, in the Proceedings of the First International Workshop on Sensor Network Protocol and Applications, Anchorage, Alaska, May 2003

[14] F. Kuhn, R. Wattenhofer, A. Zollinger,|Worst-Case optimal and average-case efficient geometric ad-hoc routing", Proceedings of the 4th ACM International Conference on Mobile Computing and Networking, Pages: 267-278, 2003.

[15] B. Chen, K. Jamieson, H. Balakrishnan, R. Morris, ISPAN: an energy-efficient coordination algorithm for topology maintenance in ad hoc wireless networks", Wireless Networks, Vol. 8, No. 5, Page(s): 481-494, September 2002

[16] W. R. Heinzelman, A. Chandrakasan, and H. Balakrishnan, "Energy- Efficient Communication Protocol for Wireless Microsensor Networks," proc. International Conference on System Sciences, Vol. 8, pp. 1-10, Jan. 2000. 